\title{
Asymptotic stress field at the tip of an inclined crack terminating to an interface
}

\author{
Liviu Marsavina ${ }^{1,2}$, Tomasz Sadowski ${ }^{1}$ \\ ${ }^{1}$ Lublin University of Technology, Faculty of Civil and Sanitary Engineering, \\ 20-618 Lublin, Nadbystrzycka 40, Poland, e-mail: t.sadowski@pollub.pl \\ 2 Politehnica University of Timisoara, Department Strength of Materials, \\ Blvd. M. Viteazu, Nr.1, Timisoara 300222, Romania, e-mail:msvina@mec.upt.ro
}

\begin{abstract}
This paper presents the numerical results for the asymptotic stress field and the fracture parameters at the tip of an inclined cracks terminating to a bi-material ceramic interface. The numerical analysis was carried out using FRANC2D/L fracture analysis code. A biaxial specimen was modeled for producing different mixed mode loads and two materials combinations of $\mathrm{Al}_{2} \mathrm{O}_{3}$ and $\mathrm{ZrO}_{2}$ were considered. The influence of the material combination and applied mixed mode load on the singularity orders, stress distributions and stress intensity factors is highlighted.
\end{abstract}

Key words: crack; bi-material interface; asymptotic stress field; stress intensity factor.

\section{Introduction}

The presence of cracks has a major impact on the reliability of advanced materials, like fiber or particle reinforced ceramic composites, ceramic interfaces, laminated ceramics. In the fabrication process of ceramic composite materials or in service interface cracks could appear in one of the constituents. These cracks growth and reach the interface and then can be deflected by the interface or can penetrate in the other constituent. Many researchers reported the presence of inclined cracks to interface. For example Kaya et al. [1] observed the deflection of an indenter induced crack when reach a zirconia - alumina interface, Fig. 1a. Tilbrook et al. [2] investigating the crack propagation path in layered and graded composite shown the influence of the interface on the crack path, Fig. 1b. In both cases the crack reach the interface at a particular angle.

Different researchers have investigated the interaction between an interface and a perpendicular or inclined crack. Zak and Williams [3] showed that the stress field singularity at the tip of a crack perpendicular to an interface or terminating at the interface is of order $r^{-\lambda}$, where $\lambda$ is the real part of the eigenvalue and depends on the elastic properties of the bi-material. Cook and Erdogan [4] used the Mellin transform method to derive the governing equation of a finite crack perpendicular to the interface and obtained the stress intensity factors. Erdogan and Biricikoglu [5] solved the problem of two bounded half planes with a crack going through the interface. Bogy [6] investigated the stress singularity of an infinite crack terminated at the interface 
with an arbitrary angle. Wang and Chen [7] used photoelasticity to determine the stress distribution and the stress intensity factors of a crack perpendicular to the interface. Lin and Mar [8], Ahmad [9] and Tan et al. [10] used finite element to analyze cracks perpendicular to bi-material in finite elastic body. Chen [11] used the body force method to determine the stress intensity factors for a normal crack terminated at a bi-material interface. Chen et al. [12] used the dislocation simulation approach in order to investigate the crack tip parameters for a crack perpendicular to an interface of a finite solid. He and Hutchinson [13] also considered cracks approaching the interface at oblique angles. Chang and $\mathrm{Xu}$ [14] presented the singular stress field and the stress intensity factors solution for an inclined crack terminating at a bi-material interface. A theoretically description of the stress singularity of an inclined crack terminating at an anisotropic bi-material interface was proposed by Lin and Sung [15]. Wang and Stahle [16] using a dislocation approach presented the complete solution of the stress field ahead of a crack approaching a bimaterial interface. They also calculate the stress intensity factor solutions and the T-stress. Liu et al. [17] determined the mixed mode stress intensity factors for a bi-material interface crack in the infinite strip configuration and in the case where both phases are fully anisotropic. Kaddouri et al. [18] and Madani et al. [19] used the finite element analysis to investigate the interaction between a crack and an interface in a ceramic/metal bi-material. They investigated the effects of the elastic properties of the two bounded materials and the crack deflection at the interface using the energy release rate. Marsavina and Sadowski [20] highlighted the effect of shielding and anti-shielding at a tip of a crack approaching a bi-material interface. A biaxial specimen subjected to mixed mode load was studied using finite element analysis.

The possibility of crack deflection or penetration when meets a bi-material interface was investigated by He et al. [21] and Marsavina and Sadowski [22] among others.

This paper presents the asymptotic stress field and the fracture parameters at the tip of an inclined cracks ended to a bi-material ceramic interface. The numerical results were obtained by considering the two materials as combinations of $\mathrm{Al}_{2} \mathrm{O}_{3}$ and $\mathrm{ZrO}_{2}$, often used in ceramic composite materials.

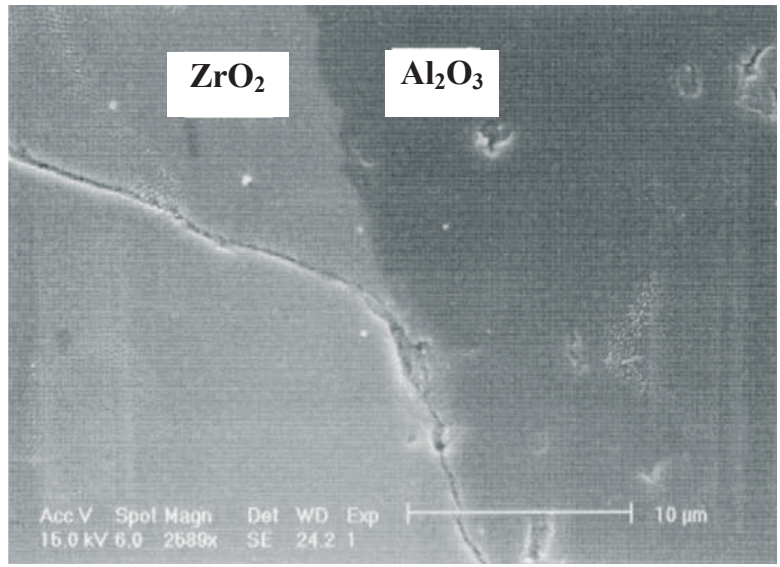

a) indentation crack deflected by interface, [1].

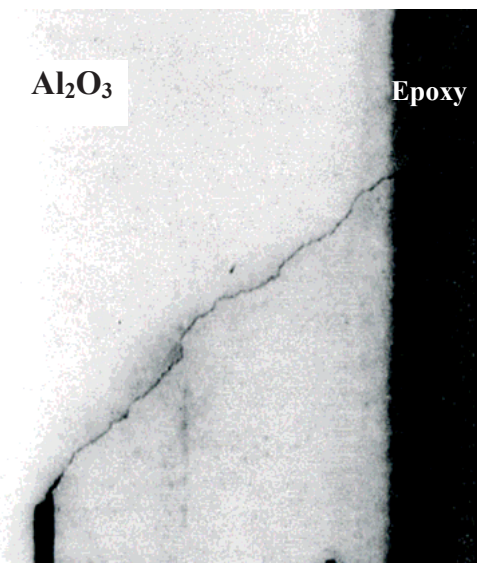

b) crack propagation path near interface, [2].

Fig. 1. Typical cases of inclined cracks to interface. 


\section{Singular stress field for an inclined crack terminating at a bi-material interface}

Considering the general case of an inclined crack terminating at the interface with angle $\theta_{0}$ (Fig. 2) the stress and displacement fields could be described by, [14]:

$$
\begin{aligned}
& \sigma_{\theta \theta, j}+i \tau_{r \theta, j}= r^{\lambda-1}\left[A_{j} \lambda^{2} e^{i(\lambda-1) \theta}+\overline{B_{j}} \lambda e^{-i(\lambda-1) \theta}+C_{j} \lambda e^{i(\lambda+1) \theta}\right]+ \\
&+r^{\bar{\lambda}-1}\left[B_{j} \bar{\lambda}^{2} e^{i(\lambda-1)}+\overline{A_{j}} \bar{\lambda} e^{-i(\bar{\lambda}-1) \theta}+D_{j} \bar{\lambda} e^{i(\bar{\lambda}+1) \theta}\right] \\
& 2 \mu_{j}\left(u_{r, j}+i u_{\theta, j}\right)=r^{\lambda}\left[A_{j} \kappa_{j} e^{i(\lambda-1) \theta}+\overline{B_{j}} \lambda e^{-i(\lambda-1) \theta}-\overline{D_{j}} e^{-i(\lambda+1) \theta}\right]+ \\
&+r^{\bar{\lambda}}\left[B_{j} \kappa_{j} e^{i(\bar{\lambda}-1) \theta}-\overline{A_{j}} \bar{\lambda} e^{-i(\bar{\lambda}-1) \theta}-\overline{C_{j}} e^{-i(\lambda+1) \theta}\right]
\end{aligned}
$$

where $(r, \theta)$ represents the polar coordinates, $\lambda$ is the stress singularity (the eigenvalue), $\bar{\lambda}$ the conjugate of $\lambda, A_{j}, B_{j}, C_{j}$ and $D_{j}$ are undetermined complex coefficients and their complex conjugates $\overline{A_{j}}, \overline{B_{j}}, \overline{C_{j}}, \overline{D_{j}}, j=1,2,3$ corresponding with region " $j$ ", and $i=\sqrt{-1}$.

The boundary conditions near the crack tip are:

$$
\begin{aligned}
& u_{r, 1}\left(r, \theta_{0}\right)=u_{r, 2}\left(r, \theta_{0}\right) \\
& u_{\theta, 1}\left(r, \theta_{0}\right)=u_{\theta, 2}\left(r, \theta_{0}\right) \\
& u_{r, 1}\left(r,-\pi+\theta_{0}\right)=u_{r, 3}\left(r,-\pi+\theta_{0}\right) \\
& u_{\theta, 1}\left(r,-\pi+\theta_{0}\right)=u_{\theta, 3}\left(r,-\pi+\theta_{0}\right) \\
& \sigma_{\theta \theta, 1}\left(r, \theta_{0}\right)=\sigma_{\theta \theta, 2}\left(r, \theta_{0}\right) \\
& \tau_{r \theta, 1}\left(r, \theta_{0}\right)=\tau_{r \theta, 2}\left(r, \theta_{0}\right) \\
& \sigma_{\theta \theta, 1}\left(r,-\pi+\theta_{0}\right)=\sigma_{\theta \theta, 3}\left(r,-\pi+\theta_{0}\right) \\
& \tau_{r \theta, 1}\left(r,-\pi+\theta_{0}\right)=\tau_{r \theta, 3}\left(r,-\pi+\theta_{0}\right) \\
& \sigma_{\theta \theta, 2}(r, \pi)=0 \\
& \tau_{r \theta, 2}(r, \pi)=0 \\
& \sigma_{\theta \theta, 3}(r,-\pi)=0 \\
& \tau_{r \theta, 3}(r,-\pi)=0
\end{aligned}
$$




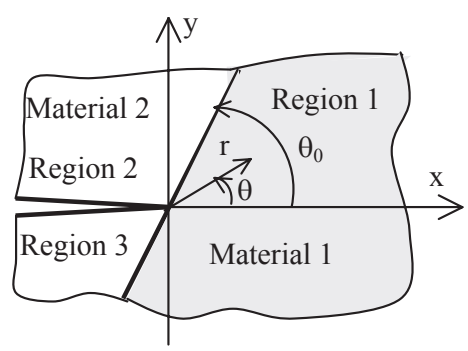

Fig. 2. Crack terminating at a bi-material interface.

Substituting eqs. (1) and (2) into eqs. (3) leads to a linear equation system with twelve undetermined coefficients: $A_{j}, \overline{B_{j}}, C_{j}$ and $\overline{D_{j}}$. From its nontrivial condition it can be obtained the following eigenequation which allows the determination of the singularity order:

$$
\begin{aligned}
& {\left[\alpha+\beta^{2}-(1-\beta)(\alpha-\beta) \lambda^{2}\left(1-\cos 2 \theta_{0}\right)+\left(1-\beta^{2}\right) \cos \pi \lambda \cos \lambda\left(\pi-2 \theta_{0}\right)\right]^{2}+} \\
& \left(1-\beta^{2}\right) \sin ^{2} \lambda\left(\pi-2 \theta_{0}\right)\left[\left(1-\beta^{2}\right) \cos ^{2} \pi \lambda+\beta^{2}-\alpha^{2}\right]=0
\end{aligned}
$$

with $\alpha$ and $\beta$ the Dundur's bi-material parameters [23]:

$$
\alpha=\frac{\mu_{1}\left(\kappa_{2}+1\right)-\mu_{2}\left(\kappa_{1}+1\right)}{\mu_{1}\left(\kappa_{2}+1\right)+\mu_{2}\left(\kappa_{1}+1\right)} \quad \text { and } \quad \beta=\frac{\mu_{1}\left(\kappa_{2}-1\right)-\mu_{2}\left(\kappa_{1}-1\right)}{\mu_{1}\left(\kappa_{2}+1\right)+\mu_{2}\left(\kappa_{1}+1\right)}
$$

where $\mu_{i}$ and $v_{i}$ are the shear modulus and Poisson's ratio of material $m(m=1,2)$, $\kappa_{m}=3-4 \nu_{m}$ for plane strain and $\kappa_{m}=\left(3-\nu_{m}\right) /\left(1+\nu_{m}\right)$ for plane stress.

Eq. (4) has normally two solutions in the interval 0 to 1 named $\lambda_{1}$ and $\lambda_{2}$, which is known as split singularities [24]. These two solutions could be complex or real numbers. It can be observed that the stress singularity depends on the material parameters and crack inclination angle.

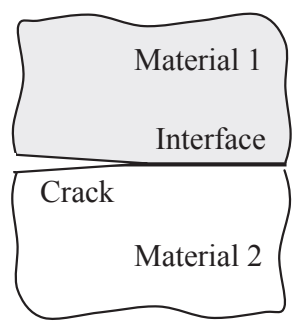

a. Interface crack

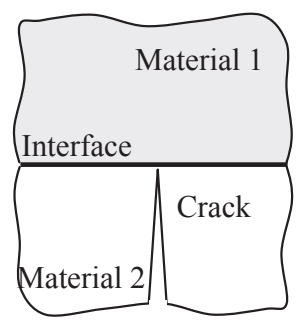

b. Crack normal to interface

Fig. 3. Particular cases of interface crack.

For $\theta_{0}=0$ or $\pm \pi$ the considered model becomes an interface crack model (Fig. 3a) and eq. (4) become:

$$
\beta^{2} \sin ^{2} \pi \lambda+\cos ^{2} \pi \lambda=0 \Rightarrow \lambda_{1,2}=\frac{1}{2} \pm i \varepsilon
$$


known as the oscillatory singularity solution for the interface crack, with the oscillatory index $\varepsilon$ :

$$
\varepsilon=\frac{1}{2 \pi} \ln \frac{1-\beta}{1+\beta}
$$

For $\theta_{0}= \pm \pi / 2$ the case of normal crack to interface (Fig. 3b) eq. (4) reduces to:

$$
\alpha+\beta^{2}-2 \lambda^{2}(\alpha-\beta)(1-\beta)+\left(1-\beta^{2}\right) \cos \pi \lambda=0
$$

according with [5] and shows that only real eigenvalues exists: $\lambda_{1}=\lambda_{2}$.

An extrapolation method could be applied for the determination of the stress intensity factors. For example if the singularity orders $\left(\lambda_{1} \neq \lambda_{2}\right)$ are real values the stress field could be expresses as:

$$
\sigma_{\theta \theta 1}+i \tau_{r \theta 1}=K_{1} r^{\lambda_{1}-1}+i K_{2} r^{\lambda_{2}-1} \quad \text { for } \theta=0,
$$

and the stress intensity factors could be found:

$$
K_{1}=\lim _{r \rightarrow 0}\left(\left.r^{1-\lambda_{1}} \sigma_{\theta \theta 1}\right|_{\theta=0}\right), \quad K_{2}=\lim _{r \rightarrow 0}\left(\left.r^{1-\lambda_{2}} \tau_{r \theta 1}\right|_{\theta=0}\right)
$$

In order to investigate the stress singularity, combinations between two ceramic materials were considered: $\mathrm{Al}_{2} \mathrm{O}_{3}$ and $\mathrm{ZrO}_{2}$ (with the mechanical properties shown in Table 1) and angles between crack and interface from $0^{\circ}$ to $90^{\circ}$ with $5^{\circ}$ increments. The results of eq. (4) are presented in Table 2 for plane strain. Only the stress singularities $\lambda_{1}$ and $\lambda_{2}$ values between 0 and 1 were considered.

Table 1. Material properties and Dundurs parameters.

\begin{tabular}{ccc}
\hline Properties/Material & $\mathrm{Al}_{2} \mathrm{O}_{3}$ & $\mathrm{ZrO}_{2}$ \\
\hline Modulus of elasticity, $[\mathrm{MPa}]$ & 400000 & 200000 \\
Poisson's ratio & 0.22 & 0.25 \\
Fracture toughness, [MPa m $\left.{ }^{0.5}\right]$ & 4.5 & 12 \\
\hline Combinations: Material 1/Material 2 & $\mathrm{ZrO}_{2} / \mathrm{Al}_{2} \mathrm{O}_{3}$ & $\mathrm{Al}_{2} \mathrm{O}_{3} / \mathrm{ZrO}_{2}$ \\
\hline Dundur's parameters & & \\
$E_{1} / E_{2},[-]$ & 0.5 & 2.0 \\
$\alpha,[-]$ & -0.333 & 0.333 \\
$\beta,[-]$ & -0.120 & 0.120 \\
\hline
\end{tabular}

Fig. 4 shows the variation of eq. (4) versus $\lambda$ for three particular cases of crack inclination angle $\theta_{0}(0 ; \pi / 4$ and $\pi / 2)$. It can be observed that eq. (4) could have two complex solution $\left(\lambda_{1,2}=\frac{1}{2} \pm i \varepsilon\right)$ for $\theta_{0}=0$, two different real solutions $\left(\lambda_{1} \neq \lambda_{2}\right)$ for $\theta_{0}=\pi / 4$ or two equal real solutions $\left(\lambda_{1}=\lambda_{2}\right)$ for $\theta_{0}=\pi / 2$. 


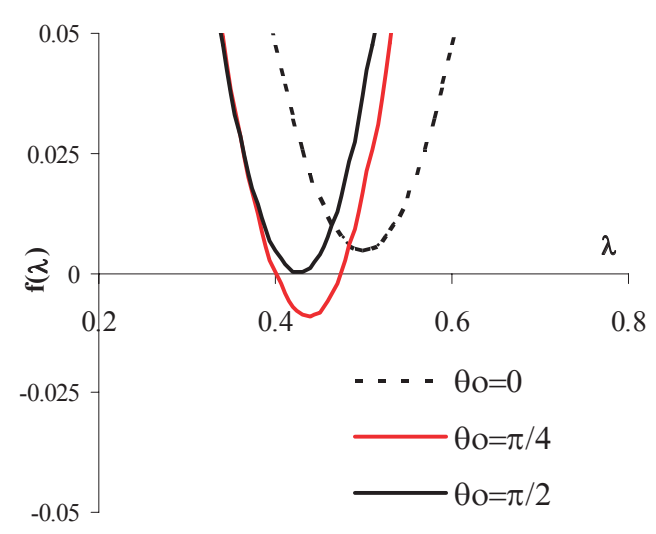

Fig. 4. Plot of $f(\lambda)$ given by eq. (4) versus $\lambda$.

Table 2 . Stress singularity $\lambda$ for plane strain.

\begin{tabular}{|c|c|c|c|c|c|c|c|c|}
\hline \multirow{3}{*}{$\begin{array}{c}\begin{array}{c}\text { Material } \\
\text { combination }\end{array} \\
\text { Singularities } \\
\text { Angle } \\
\theta_{0}[\mathrm{deg}]\end{array}$} & \multicolumn{4}{|c|}{$E_{1} / E_{2}=0.5$} & \multicolumn{4}{|c|}{$E_{1} / E_{2}=2.0$} \\
\hline & \multicolumn{2}{|c|}{$\lambda_{1}$} & \multicolumn{2}{|c|}{$\lambda_{2}$} & \multicolumn{2}{|c|}{$\lambda_{1}$} & \multicolumn{2}{|c|}{$\lambda_{2}$} \\
\hline & $\operatorname{Re}\left(\lambda_{1}\right)$ & $\operatorname{Im}\left(\lambda_{1}\right)$ & $\operatorname{Re}\left(\lambda_{2}\right)$ & $\operatorname{Im}\left(\lambda_{2}\right)$ & $\operatorname{Re}\left(\lambda_{1}\right)$ & $\operatorname{Im}\left(\lambda_{1}\right)$ & $\operatorname{Re}\left(\lambda_{2}\right)$ & $\operatorname{Im}\left(\lambda_{2}\right)$ \\
\hline 0 & 0.5000 & 0.0320 & 0.5000 & 0.0320 & 0.5000 & 0.0320 & 0.5000 & 0.0320 \\
\hline 5 & 0.5070 & 0.0319 & 0.5070 & 0.0319 & 0.4873 & 0.0288 & 0.4873 & 0.0288 \\
\hline 10 & 0.5142 & 0.0307 & 0.5142 & 0.0307 & 0.4764 & 0.0213 & 0.4764 & 0.0213 \\
\hline 15 & 0.5217 & 0.0280 & 0.5217 & 0.0280 & 0.4672 & 0.0014 & 0.4672 & 0.0014 \\
\hline 20 & 0.5293 & 0.0235 & 0.5293 & 0.0235 & 0.4806 & & 0.4387 & \\
\hline 25 & 0.5367 & 0.0158 & 0.5367 & 0.0158 & 0.4820 & & 0.4248 & \\
\hline 30 & 0.5538 & & 0.5340 & & 0.4816 & & 0.4150 & \\
\hline 35 & 0.5720 & & 0.5292 & & 0.4801 & & 0.4080 & \\
\hline 40 & 0.5844 & & 0.5285 & & 0.4778 & & 0.4032 & \\
\hline 45 & 0.5933 & & 0.5292 & & 0.4749 & & 0.4003 & \\
\hline 50 & 0.5988 & & 0.5309 & & 0.4713 & & 0.3989 & \\
\hline 55 & 0.6011 & & 0.5333 & & 0.4671 & & 0.3990 & \\
\hline 60 & 0.6004 & & 0.5363 & & 0.4623 & & 0.4004 & \\
\hline 65 & 0.5973 & & 0.5400 & & 0.4571 & & 0.4028 & \\
\hline 70 & 0.5925 & & 0.5442 & & 0.4513 & & 0.4062 & \\
\hline 75 & 0.5866 & & 0.5491 & & 0.4453 & & 0.4104 & \\
\hline 80 & 0.5801 & & 0.5544 & & 0.4391 & & 0.4153 & \\
\hline 85 & 0.5733 & & 0.5603 & & 0.4329 & & 0.4208 & \\
\hline 90 & 0.5667 & & 0.5667 & & 0.4267 & & 0.4267 & \\
\hline
\end{tabular}

From Table 2 and Fig. 4 it can be observed that depending on angle $\theta_{0}$ we can have a complex or oscillatory solution (for low angles $\theta_{0}=0^{\circ}$ representing an interface crack), multiple singularities and double root singularity (for $\theta_{0}=90^{\circ}$ ). It can be observed that higher singularities than 0.5 were obtained when the crack is in the $\mathrm{ZrO}_{2}\left(E_{1} / E_{2}=0.5\right)$, and lower values for $E_{1} / E_{2}=2.0$ when the crack is in the stiffer material. 


\section{The asymptotic stress field for an inclined crack terminating to interface}

\subsection{Problem description}

The biaxial specimen with an inclined crack at $45^{\circ}$ has been successfully used to growth mixed mode cracks [25] and to investigate the singular stress field in mixed mode conditions. In contrast with the specimens containing inclined cracks in monoaxial tension, this type of specimen has the advantage of creating different type of mixed modes at the crack tip on the same geometry, only by changing the applied loads on the two axes, Fig. 5.

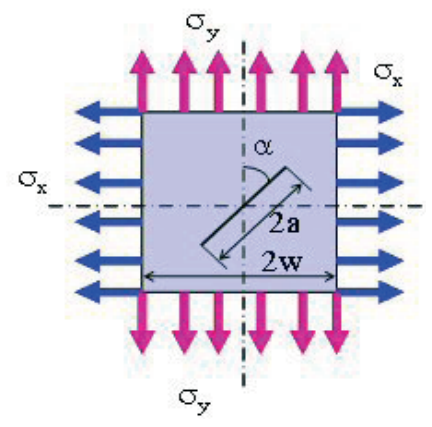

Fig. 5. Biaxial specimen.

In order to study the effect of the biaxial load on the asymptotic stress field a quarter of a biaxial specimen with an interface was numerically investigated with FRANC2DL code, [26]. Plane strain conditions were considered and the model dimensions were $w=50 \mathrm{~mm}$ and $a=70.71 \mathrm{~mm}$. The materials considered for the analysis were combinations between $\mathrm{Al}_{2} \mathrm{O}_{3}$ and $\mathrm{ZrO}_{2}$, Table 1. Quadratic isoparametric elements were used for the model with a refined mesh near to crack tip, Fig. 6. Eight singular elements were placed around the crack tip as a common technique to model the stress singularity. The mesh consists 11754 elements connected in 34353 nodes.

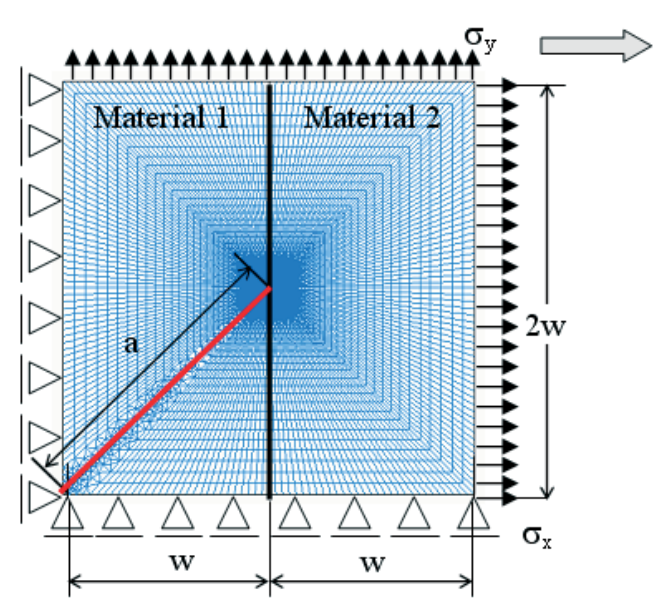

a) Mesh and boundary conditions.

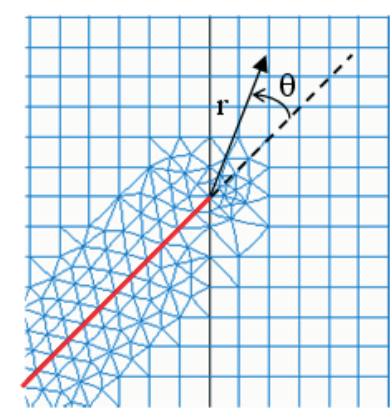

b) Crack detail.

Fig. 6. Mesh, boundary conditions and crack detail for the FEM model. 
The model was loaded with five different combinations of the applied stresses $\sigma_{x}$ and $\sigma_{y}\left(k=\sigma_{x} / \sigma_{y}\right)$ in order to produce mixed modes from pure Mode I $(k=1)$ to pure Mode II $(k=-1)$. The symmetric boundary conditions were imposed. The polar coordinate system $(r, \theta)$ was considered with the origin at the crack tip, Fig. 6b.

A convergence study for the asymptotic analysis and mesh density was prior carried on for a crack perpendicular to an interface in a sandwich plate [27]. The obtained numerical results agrees with the analytical solution of the asymptotic stress field, for example the singularity orders were between $0.8 \%$ to the analytical solution. The same analysis parameters were considered like: mesh density at the crack tip (the smallest element size at the crack tip $10^{-4} a$ ) and intervals for asymptotic analysis.

\subsection{Split singularities}

The singularity orders $\lambda_{1}$ and $\lambda_{2}$ were determined numerically from the asymptotic stress field on a direction $\theta=-15^{0}$ as the slope $\left(1-\lambda_{1,2}\right)$ of the $\log \left(\sigma_{\theta \theta} / \sigma_{y}\right)$ versus $\log (r / a)$, respectively $\log \left(\tau_{r \theta} / \sigma_{y}\right)$. The main steps of this asymptotic analysis are summarized below:

- the numerical results for $\sigma_{\theta \theta}$ (respectively for $\tau_{r \theta}$ ) were collected on a path with $\theta=-15^{\circ}$, Fig. 7a;

- the stresses were normalized to the applied load on $y$ direction, and were plotted on logarithmic scale, fig. 7b;

- from a linear interpolation the quantity $1-\lambda_{1,2}$ was obtained as the slope of the trendline, and then singularity orders $\lambda_{1}$ and $\lambda_{2}$ were determined. It can be observed that the coefficient of determination for the regression analysis $\left(R^{2}-\right.$ value) approach 1 which indicates a good linear fit of numerical data.

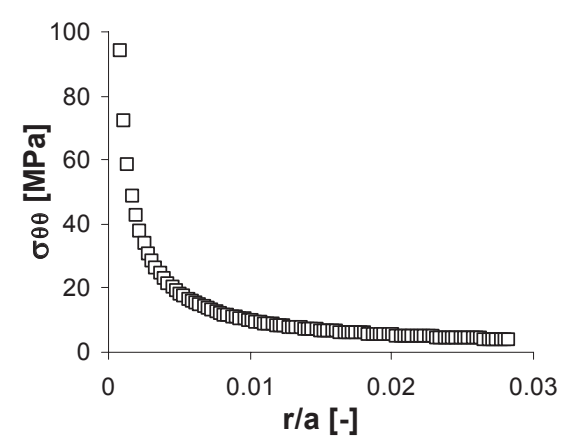

a) Numerical results of $\sigma_{\theta \theta}$ versus $r / a$.

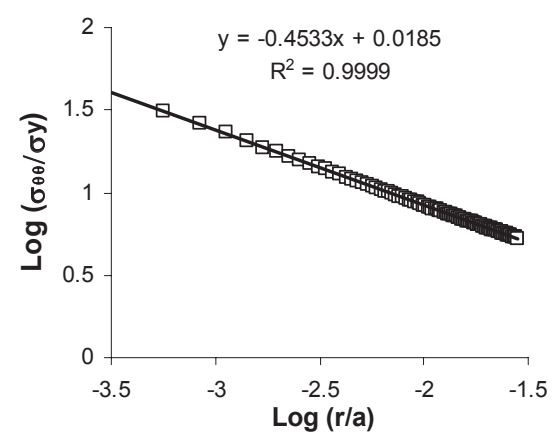

b) Linear interpolation for logarithmic plot.

Fig. 7. The determination of the singularity orders, case of material combination $E_{1} / E_{2}=0.5$ and load combination $k=1$.

The obtained results of singularity orders are presented in Table 3.

Table 3. Singularity orders for different combinations of mixed mode loading.

\begin{tabular}{ccccccc}
\hline Material combination & $k$ & 1.0 & 0.50 & 0.0 & -0.5 & -1.0 \\
\hline \multirow{2}{*}{$E_{1} / E_{2}=0.5$} & $\lambda_{1}$ & 0.5467 & 0.5566 & 0.5730 & 0.5995 & 0.6501 \\
& $\lambda_{2}$ & 0.8342 & 0.8321 & 0.6342 & 0.6457 & 0.5942 \\
\hline \multirow{2}{*}{$E_{1} / E_{2}=2.0$} & $\lambda_{1}$ & 0.4587 & 0.4475 & 0.4296 & 0.4086 & 0.3756 \\
& $\lambda_{2}$ & 0.1955 & 0.2250 & 0.3710 & 0.4039 & 0.4186 \\
\hline
\end{tabular}


The results from Table 3 show that the singularity orders vary significantly with applied load combination and material properties. It can be observed that the singularity order $\lambda_{1}$ increase with the increasing of mode II load (decreasing k) for $E_{1} / E_{2}=0.5$. In contrary for the material combination with $E_{1} / E_{2}=2.0$ the singularity order decrease with decreasing $k$. The singularity order $\lambda_{2}$ decrease with decreasing $k$ for material combination with $E_{1} / E_{2}=0.5$ and increase with decreasing $k$ for $E_{1} / E_{2}=2.0$.

\subsection{Asymptotic stress field - radial variations}

Fig. 8 presents the radial variation of the stresses $\sigma_{\theta \theta}$ (normalized to applied stress in $y$ direction) in logarithmic coordinates for the two considered materials combinations on a radial path with $\theta=-15^{0}$ and for $0<r / a<0.028$. For the same radial parameters $(\theta, r)$ Fig. 9 presents the distribution of tangential stress $\tau_{r \theta}$. For both materials combinations and applied load mixities $k$ the radial variations are linear on the log-log plot. The slope of the lines in Fig. 8 are equal with $1-\lambda_{1}$, while in Fig. 9 the slopes are $1-\lambda_{2}$.

a) $E_{1} / E_{2}=0.5$

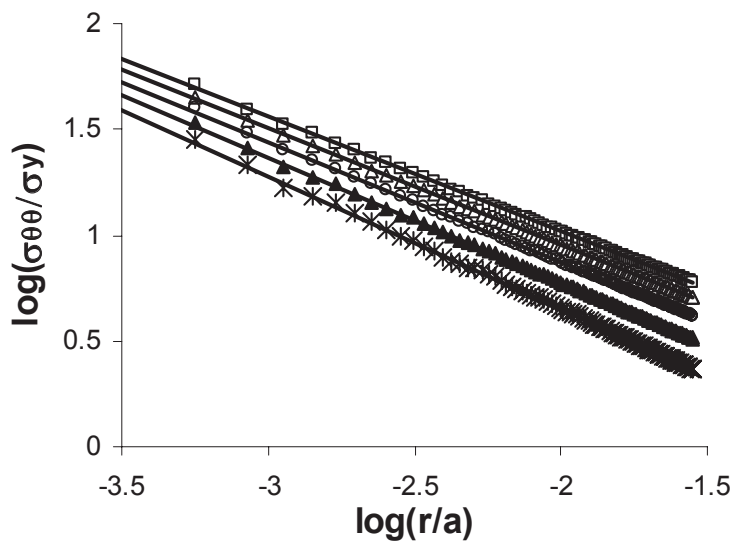

b) $E_{1} / E_{2}=2.0$

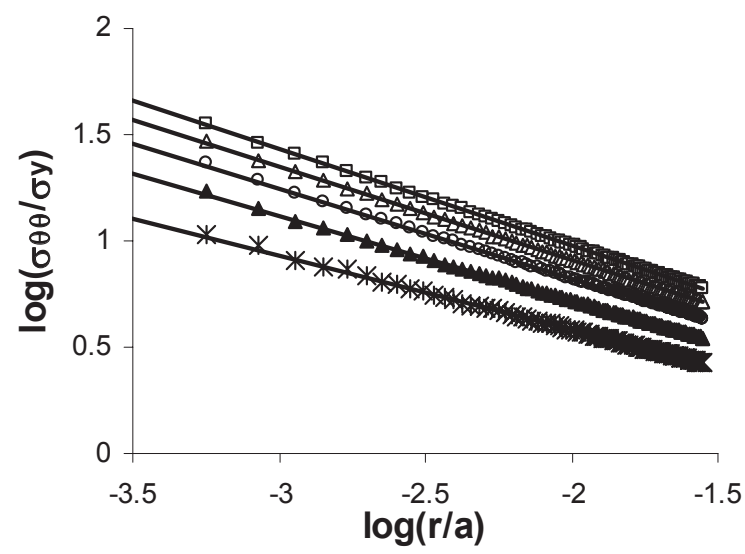

Fig. 8. Logarithmic distribution of $\sigma_{\theta \theta}$ stresses on a radial direction $\left(\theta=-15^{\circ}\right)$, $\square \mathrm{k}=1.0, \Delta \mathrm{k}=0.5$, $\circ \mathrm{k}=0.0, \boldsymbol{\Delta} \mathrm{k}=-0.5, * \mathrm{k}=-1.0$. 
a) $E_{1} / E_{2}=0.5$

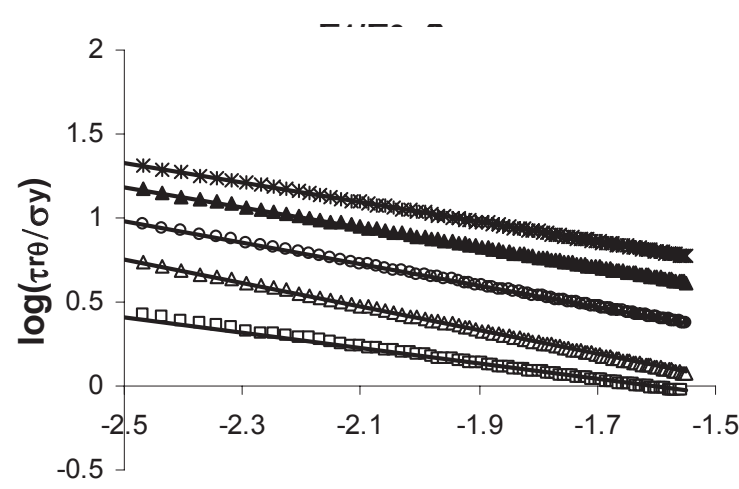

\section{$\log (\mathbf{r} / \mathrm{a})$}

b) $E_{1} / E_{2}=2.0$

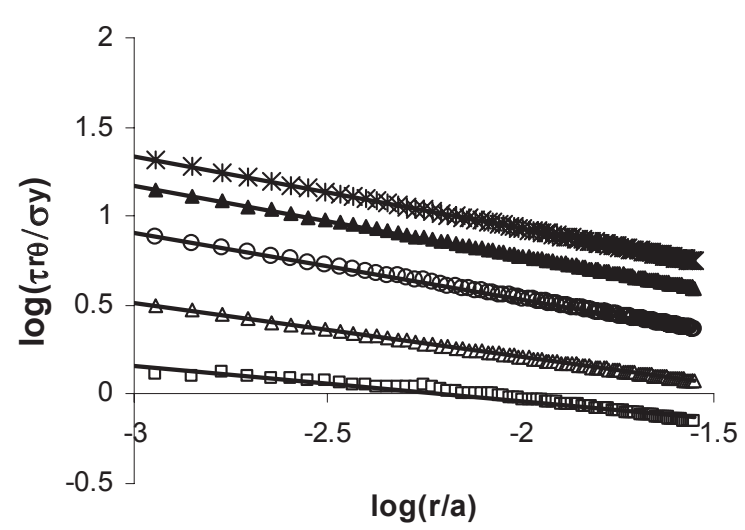

Fig. 9. Logarithmic distribution of $\tau_{r \theta}$ stresses on a radial direction $\left(\theta=-15^{\circ}\right)$. $\square \mathrm{k}=1.0, \Delta \mathrm{k}=0.5$, $\circ \mathrm{k}=0.0, \boldsymbol{\Delta} \mathrm{k}=-0.5, * \mathrm{k}=-1.0$.

For both material combinations it can be observed that the singularity orders are sensitive with the material combination and load parameter $k$. As we expect maximum circumferential stresses $\sigma_{\theta \theta}$ were obtained for loads with $k=1$ (mode I) and minimum values for $k=-1$ (mode II), and vice versa for tangential stresses $\tau_{r \theta}$. Comparing the value of stresses between the two material combinations it can be observed that for the same loading case $k$ higher values for the stresses $\sigma_{\theta \theta}$ and $\tau_{r \theta}$ were obtained when the crack was in the compliant material $\left(E_{1} / E_{2}=0.5\right)$.

\subsection{Asymptotic stress field - circumferential variations}

The circumferential stress distributions are plotted at a distance $r / a=0.01$ from the crack tip, in order to be in the singularity zone. The circumferential variation of $\sigma_{\theta \theta}$ normalized to the applied stress in y direction $\sigma_{y}$ is plotted in Fig. 10, and for tangential stress $\tau_{r \theta}$ in Fig. 11 for both material combinations. 
a) $E_{1} / E_{2}=0.5$

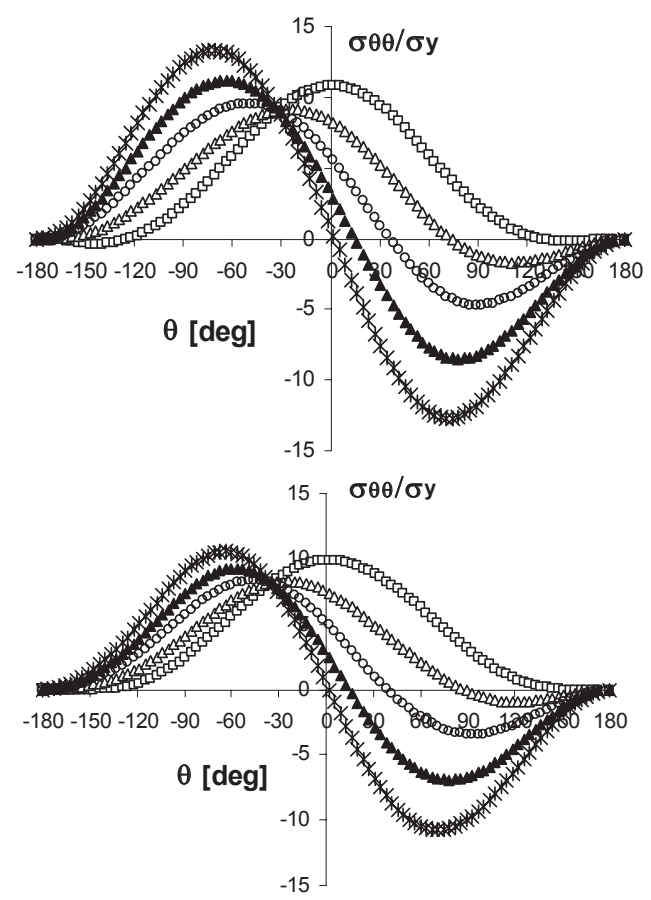

Fig. 10. Circumferential distribution of $\sigma_{\theta \theta}$ stresses at distance $r / a=0.01, \square \mathrm{k}=1.0, \Delta \mathrm{k}=0.5$, $\circ \mathrm{k}=0.0, \boldsymbol{\Delta} \mathrm{k}=-0.5, * \mathrm{k}=-1.0$.

a) $E_{1} / E_{2}=0.5$
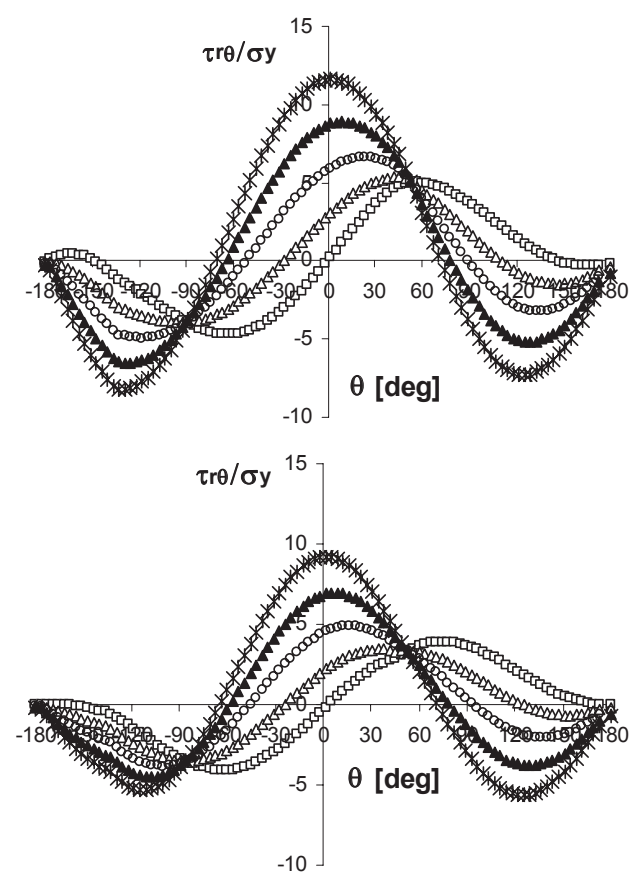

Fig.11 Circumferential distribution of $\tau_{r \theta}$ stresses at distance $r / a=0.01, \square \mathrm{k}=1.0, \Delta \mathrm{k}=0.5$, $\circ \mathrm{k}=0.0, \boldsymbol{\Delta} \mathrm{k}=-0.5, * \mathrm{k}=-1.0$. 
It can be observed that the distribution of $\sigma_{\theta \theta}$ is changing from symmetric for $k=1$ to anti-symmetric for $k=-1$, and vice versa for $\tau_{r \theta}$ distribution. The maximum values for the normal stress $\sigma_{\theta \theta}$ on the crack direction $\left(\theta=0^{\circ}\right)$ were obtained for $k=1$ (mode I) and the minimum for $k=-1$ (mode II) Fig. 10, as we expect. Fig. 11 shows that the maximum circumferential stress $\tau_{r \theta}$ on the crack line $\left(\theta=0^{0}\right)$ was obtained for $k=-1$ (load in pure mode II) and is 0 for $k=1$ (load in pure Mode I). The maximum values of the tangential stress $\tau_{r \theta}$ moves from $\theta=0^{0}$ for $k=-1$ to $\theta=74.5^{\circ}$ for $k=1$. The stress results from Figs. 10 and 11 confirm the stress free boundary conditions on the crack faces $\left(\theta= \pm 180^{\circ}\right)$.

\subsection{Stress intensity factors}

The determination of the Stress Intensity Factors (SIF) was performed using eq. (10), by extrapolating at the crack tip the asymptotic results of $\sigma_{\theta \theta}$ and $\tau_{r \theta}$ for a path with $\theta=0^{\circ}$. Fig. 12 shows the determination of the stress intensity factors from numerical results for material combination with $E_{1} / E_{2}=0.5$ and mixed mode case with $\mathrm{k}=0.0$. The obtained values of SIF's are $K_{1}=4.273 \mathrm{MPa} \mathrm{m}^{1-\lambda 1}$ and $K_{2}=4.0 \mathrm{MPa} \mathrm{m}^{1-\lambda 2}$.

Taking into account the dimensions for the SIF's [MPa $\left.\mathrm{m}^{1-\lambda}\right]$ it is hard to make comparisons between obtained results. Usually, normalized stress intensity factors are used for example in [11], [28]:

$$
f_{1}=\frac{K_{1}}{\sigma_{j} a^{1-\lambda_{1}}}, \quad f_{2}=\frac{K_{2}}{\sigma_{j} a^{1-\lambda_{2}}}
$$

The normalized stress intensity factor results are shown in Fig. 13. The computed values of the normalised SIF's cover entire domain from pure mode I $(k=1)$ to pure mode II $(k=-1)$. Higher values for normalised SIF's were obtained for the case when the crack is in the compliant material for the same load combination.

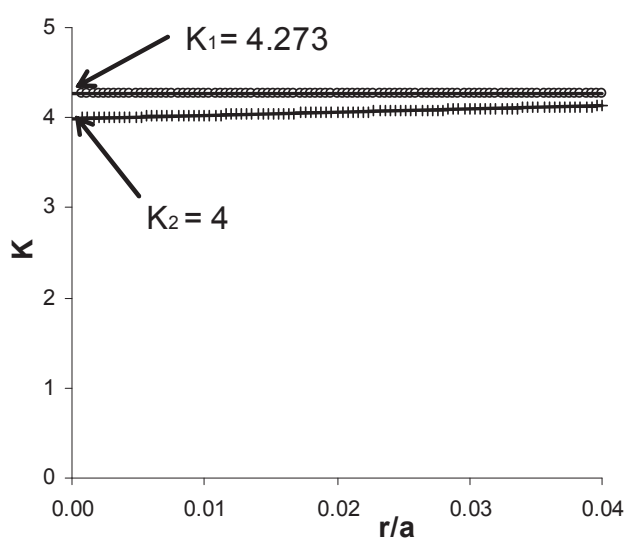

Fig. 12. SIF determination by extrapolation, $\circ \sigma_{\theta \theta},+\tau_{r \theta}$.

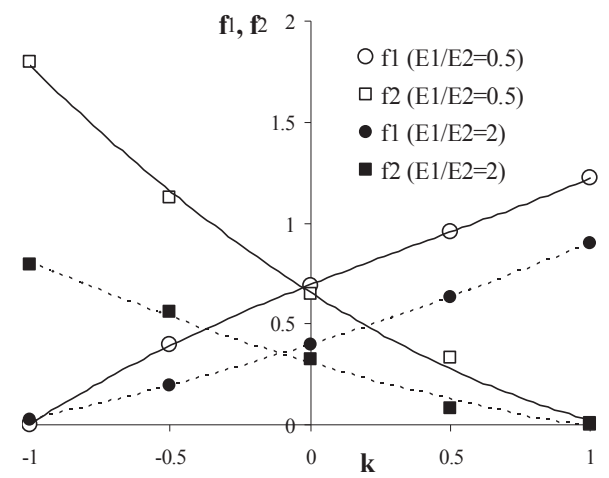

Fig. 13. Non-dimensional stress intensity factors. 
Asymptotic stress field at the tip of an inclined crack terminating to an interface. 123

\section{Conclusions}

For an inclined crack to a bi-material interface it was shown that the singular stress field could be expressed as a linear superposition of the modes, usually of unequal exponents.

For two material combinations by solving the eigenequation different types of singularities were obtained: complex, multiple or single depending on crack angle $\theta_{0}$ and on material combinations. The singularity order $0<\lambda<0.5$ when the crack is in the stiffer material and meets the compliant ones, and $0.5<\lambda<1$ when vice versa.

For an $45^{\circ}$ inclined crack terminating on a bi-material interface the asymptotic stress field was numerically investigated. Five loading combinations were considered from pure Mode I to pure Mode II. The split singularities of the asymptotic stress field were numerically determined.

The stress intensity factors were estimated by extrapolation technique, based on the numerical results for the asymptotic stress field.

The effect of the bi-axial load on the asymptotic stress field and on the stress intensity factors for an inclined crack terminated on the interface was highlighted.

\section{Acknowledgements}

The authors gratefully acknowledge the support of Marie Curie Transfer of Knowledge project MTKD-CT-2004-014058.

\section{References}

[1] Kaya C., Butler E.G., Lewis M.H., Co-extrusion of Al2O3/ZrO2 bi-phase high temperature ceramics with fine scale aligned microstructures, Journal of the European Ceramic Society 23, (2003), pp. 935-942.

[2] Tilbrook M. T., Rozenburg K., Steffler E. D., Rutgers L, Hoffman M., Crack propagation paths in layered, graded composites, Composites: Part B 37, (2006), pp. 490-498.

[3] Zak A.R., Williams M.L., Crack point stress singularities at a bi-material interface, J. Appl. Mech, Volume: 30, (1963), pp. 142-143.

[4] Cook T.S., Erdogan F., Stress in bonded materials with a crack perpendicular to the interface, Int. J. Eng. Sci., Volume: 10, (1972), pp. 677-697.

[5] Erdogan F., Biricikoglu V., Two bonded half planes with a crack going through the interface, Int. J. Engng. Sci., Volume: 11, (1973), pp. 745-766.

[6] Bogy D.B., On the plane elastic problem of a loaded crack terminating a material interface, J. Int. Fract., Volume: 38, (1971), pp. 911-918.

[7] Wang W.C., Chen J.T., Theoretical and experimental re-examination of a crack at a bimaterial interface, J. Strain Anal., Volume: 28, (1993), pp. 53-61.

[8] Lin K.Y., Mar J.W., Finite element analysis of stress intensity factors for crack at a bimaterial interface, Int. J. Fract., Volume: 12, (1976), pp. 451-531.

[9] Ahmad J., A micromechanics analysis of cracks in unidirectional fibre composite, J. Appl. Mech., Volume: 58, (1991), pp. 964-972.

[10] Tan M., Meguid S.A., Dynamic analysis of cracks perpendicular to bimaterial interfaces using new singular finite element, Finite Elements in Analysis and Design, 22, (1996), pp. 69-83.

[11] Chen D.H., A crack normal to and terminating at a bimaterial interface, Engng. Fract. Mech., Volume: 19, (1994), pp. 517-532. 
[12] Chen S.H., Wang T.C., Kao - Walter S., A crack perpendicular to the bi-material interface in finite solid, Int. J. Solids Struct, 40, (2003), pp. 2731-2755.

[13] He M.Y., Hutchinson J.W., Crack deflection at an interface between dissimilar elastic materials, Int. J. Solids Struct., Volume: 25, (1993), pp. 1053-1067.

[14] Chang J., Xu J.-Q., The singular stress field and stress intensity factors of a crack terminating at a bimaterial interface, Int. J. Mechanical Sciences, 49, (2007), pp. 888-897.

[15] Lin Y.Y., Sung J.C., Singularities of an inclined crack terminating at an anisotropic biomaterial interface, Int. J. Solids Struct, 38, (1997), pp. 3727-3754.

[16] Wang T.C., Stahle P., Stress state in front of a crack perpendicular to bi-material interface, Engng. Fract. Mech., Volume: 4, (1998), pp. 471-485.

[17] Liu L., Kardomateas G. A., Holmes J. W., Mixed - mode stress intensity factors for a crack in an anisotropic bi-material strip, Int. J. Solids Struct., 41, (2004), pp. 3095-3017.

[18] Kaddouri K., Belhouari M., Bachir Bouiadjra B., Serier B., Finite element analysis of crack perpendicular to bi-material interface: Case of couple ceramic-metal, Comput. Mater. Sci., 35, (2006), pp. 53-60.

[19] Madani K., Belhouari M., Bachir Bouiadjra B., Serier B., Benguediab M., Crack deflection at an interface of alumina/metal joint: A numerical analysis, Comput. Mater. Sci., 35, (2007), pp. 625-630.

[20] Marsavina L., Sadowski T., Fracture parameters at bi-material ceramic interfaces under bi-axial state of stress, Proceedings of IWCMM 17, Paris, 2007, p. 45-46.

[21] He M.Y., Hsueh C.H., Becher P.F., Deflection versus penetration of a wedge-load crack: effects of branch-crack length and penetrated-layer width, Composites: Part B, 31, (2000), pp.299-308.

[22] Marsavina L., Sadowski T., Effect of biaxial load on crack deflection/penetration at bi-material ceramic interface, Int. J. Fracture (2008), OnLine First, DOI: 10.1007/s10704-0089181-y.

[23] Dundurs J., Effect of elastic constants on stress in a composite under plane deformation, J. Compos. Mater., Volume: 1, (1969), pp. 310-322.

[24] Zang Z., Suo Z., Split singularities and the competition between crack penetration and debond at a bimaterial interface, Int. J. Solids Struct., 44, (2007), p. 4559-4573.

[25] Bold P. E, Brown M. W., Allen R.J., Shear Mode crack growth and rolling contact fatigue, Wear, 144, (1991), pp. 307-317.

[26] Iesulauro, E., FRANC2D/L a Crack Propagation simulator for plane layered materials, Cornell University, Ithaca, 2002.

[27] Marsavina L., Sadowski T., The influence of the interface on fracture parameters, Proceedings of the AIQ - ICF Conference, Alger 2008 (in press).

[28] Murakami Y., Stress intensity factors handbook, Vol. I, Pergamon Press, Oxford, 1987. 